\title{
Older female relatives of Covid-19 patients have an un- satisfactory perception of emergency room performance by clinical staff
}

\author{
Tiziana Ciarambino ${ }^{\mathrm{a}}$, Luigia Palmiero ${ }^{\mathrm{b}}$, Rosario Bottone ${ }^{\mathrm{a}}$, Federico Schettini ${ }^{\mathrm{a}}$, Luigi Elio Adinolfi ${ }^{\mathrm{b}}$, \\ Mauro Giordano ${ }^{\mathrm{b}, *}$ \\ ${ }^{a}$ Department of Internal Medicine, Hospital of Marcianise, ASL Caserta, Italy. \\ ${ }^{b}$ Department of Advanced Medical and Surgical Sciences, University of Campania "L. Vanvitelli", 80138 Naples, Italy.
}

\begin{abstract}
Satisfaction levels in relatives of suspected Covid-19 cases during hospital admission to an emergency department have not previously been reported. A prospective observational study was conducted using the Communication Attitude Test (CAT-T) questionnaire to describe educational level, age, and gender differences in 1201 relatives of suspected Covid-19 patients admitted to an emergency room between 21 February and 31 December 2020. Increased CAT-T scores, indicating low levels of satisfaction, were observed in older female relatives with low education levels $(\mathrm{p}<0.01)$. These observations suggest that lack of accessible information during the Covid-19 pandemic can be a key aspect of anxiety not only in patients but for relatives, especially older women, and emphasizes the need for proactive communication.
\end{abstract}

Keywords: COVID-19 pandemic, satisfaction of relatives, age, gender, education, communication

\section{Case Report and Discussion}

No studies have reported the satisfaction level in relatives of suspected Covid-19 cases during hospital admission to an emergency department. A prospective observational study was conducted at the Marcianise Hospital, ASL Caserta, Italy. The Ethics Committees Campania Nord approved it. All participants signed a consent form to be interviewed. A total of 1500 relatives of suspected Covid-19 patients were enrolled between February and December 2020. The database included demographic characteristics, educational level, clinical outcome, age, and gender, with 148 relatives less than 18 years old and 151 patients without information on education level being excluded. Educational level was classified as high (a bachelor's degree or higher), medium (graduated from high school or middle school), or low (graduated from elementary school

\footnotetext{
* Corresponding author: Mauro Giordano, MD. PhD.

Mailing address: Department of Advanced Medical and Surgical Sciences, University of Campania "L. Vanvitelli", P.zza L. Miraglia, 2-80138 Naples, Italy.

Email: mauro.giordano@unicampania.it

Received: 19 May 2021 / Accepted: 04 June 2021
}

or no education). The questionnaire Communication Attitude Test (CAT-T) [1] included clinical history, age, sex, nationality, educational level, and triage code. Additional exclusion criteria included relatives with neuro-psychiatric diseases and psychological instability. A descriptive analysis was used to compare the demographic and clinical characteristics of relatives with low, medium, and high levels of education. This included age, sex, medical history, and health behaviors. The chi square test was used to compare categorical variables with higher scores indicating decreasing satisfaction.

Results showed that the CAT-T score was significantly higher in females compared to males $(\mathrm{p}<0.001)$, and was significantly increased in relatives 65 years and older compared to under 65 years old $(\mathrm{p}<0.01)$. When compared by educational level and age, the CAT-T score was significantly increased in older relatives with a low educational level ( $p<0.01)$. These observations suggest age, gender differences and educational level play a role in the satisfaction of relatives of suspected Covid-19 cases seen in an emergency room setting. In particular, older, less educated female relatives were likely to have an unsatisfactory perception of the way the Covid-19 case was handled in the emergency room. Interestingly, a recent study showed that male and younger Covid-19 patients were more suspicious of possible clinical mistakes, but that the satisfaction level of patients increased with the level of education [2]. It is 
therefore crucial to provide guidance on how to communicate with patients and relatives, since lack of accessible information during the Covid-19 pandemic can be a key aspect of anxiety. In this regard, increased awareness of clinicians and emergency room staff to more fully communicate aspects of Covid-19 illness not only to patients but also to close relatives would help increase satisfaction, decrease anxiety, and decrease potential for complaints [3, 4].

\section{Declarations}

Conflict of interest: All authors declared that there are no conflicts of interest.

\section{References}

1. Makoul G, Krupat E, Chang C H. Measuring patient views of physician communication skills: development and testing of the Communication Assessment Tool. Patient education and counseling, 2007, 67(3): 333-342.

2. Mazor K M, Simon S R, Yood R A, et al. Health plan members' views on forgiving medical errors. Am J Manag Care, 2005, 11(1): 49-52.

3. Taylor D M D, Wolfe R, Cameron P A. Complaints from emergency department patients largely result from treatment and communication problems. Emergency Medicine, 2002, 14(1): 43-49.

4. Slade D, Manidis M, McGregor J, et al. Communicating in hospital emergency departments. Springer, 2015.

Cite this article as: Tiziana C, Luigia P, Rosario B, et al. Older female relatives of Covid-19 patients have an unsatisfactory perception of emergency room performance by clinical staff[J]. Aging Pathobiology and Therapeutics, 2021, 3(2): 37-38. 